\title{
The influence of convection on the behaviour of a ventilated BIPV module: A sensitivity analysis
}

\author{
Juliana E. Gonçalves ${ }^{1,2}$, Twan van Hooff ${ }^{1,3}$, Dirk Saelens ${ }^{1,2}$ \\ ${ }^{1}$ Department of Civil Engineering, Building Physics Section, KU Leuven, Leuven, Belgium \\ ${ }^{2}$ EnergyVille, Genk, Belgium \\ ${ }^{3}$ Department of the Built Environment, unit Building Physics and Services, \\ Eindhoven University of Technology, Eindhoven, The Netherlands
}

\begin{abstract}
Building integrated photovoltaic (BIPV) systems are considered as a promising technology to achieve low energy buildings. Although a significant body of literature is available on BIPV modelling and validation, sensitivity analysis (SA) has not been extensively explored in a systematic way. SA provides insight on how the model responds to variations on its inputs, being thus an integral part of modelling approaches. In this paper, a variance-based SA is performed to identify the dominant parameters on the performance of ventilated facade-BIPV modules, evaluated in terms of power output and cell temperature. This work uses a multi-physics BIPV model previously validated with experimental data from two BIPV setups. Convective heat transfer coefficients (CHTCs) and parameters associated to ventilation are the inputs to the SA. Results show that the heat transfer to the exterior environment and to the cavity interior have the largest influence on both power and cell temperature. Furthermore, the effects of interactions among the inputs have been found to account for most of the output variability.
\end{abstract}

\section{Introduction}

Renewable energy sources such as photovoltaic (PV) systems are key to achieve low energy buildings and reduce the impact of the building sector on the climate. The conventional application of PV modules in the built environment requires the installation of a supporting structure, normally on the existing roof, to which the PV modules are attached, the so-called building applied photovoltaics (BAPV). For aesthetic reasons, BAPV applications are not common in facades. Alternatively, PV modules can be integrated into the building envelope, replacing convectional building components and eliminating the need for the mounting structure. This concept is known as building integrated photovoltaics (BIPV).

Besides producing electricity, BIPVs provide at least one envelope function, such as insulation, weather barrier, or sun shading (Osseweijer et al. (2018)). In particular, facade integration has been considered a promising BIPV application as it complements the surface area available in rooftops (Osseweijer et al. (2018)) and provides a balanced power profile over the day, especially in the summer (Brito et al. (2017)). Furthermore, in terms of aesthetics, the integrated concept has the potential to facilitate the assimilation of BIPVs in the building design, often considered as an advantage over BAPVs (Osseweijer et al. (2018) Saretta et al. (2018)).

BIPV applications typically experience higher temperatures as the BIPV module is part of the building envelope instead of being exposed to the ambient conditions on both front and back surfaces, leading to reduced heat transfer. After shading, high operating temperatures are the second major cause of reduced energy yield, since the (BI)PV behaviour is temperature-dependent (Thevenard (2005)). With a temperature coefficient for the maximum power of about $-0.4 \% /{ }^{\circ} \mathrm{C}$, crystalline silicon (c-Si) cells experience a power reduction of $8 \%$ when operating at $45{ }^{\circ} \mathrm{C}$ (Thevenard (2005)).

The use of ventilated cavities has been explored by different authors to reduce the operating temperature in both BIPV and BAPVs. In a review study, Agathokleous and Kalogirou (2016) highlight that, on the one hand, an important research effort has been devoted to mechanically ventilated BIPVs, even though mechanical ventilation has several disadvantages, associated to the use of a fan to create the air circulation (reliability, noise, energy consumption and additional investment). On the other hand, natural ventilation has not been explored extensively due to the complex interaction between buoyant and winddriven flows, despite the advantage of exploiting these natural forces to drive the airflow.

In fact, natural ventilation of cavities in the built environment involves a strong coupling between the flow around the building and the airflow inside the cavity. At the cavity level, the pressure loss characteristics will determine the resulting airflow (Langmans and Roels (2015)). Cavity losses depend on the cavity geometry and material properties as well as on the airflow regime. 
At the building envelope level, the distribution of heat transfer coefficients and (wind) pressure coefficients will dictate heat transfer and flow conditions. Montazeri et al. (2015) have demonstrated that external convective heat transfer coefficients resulting from wind effects $\left(\mathrm{eCHTC}_{w}\right)$ not only vary over the building envelope, but also depend on the building geometry. The degree of exposure/sheltering in urban clusters also affects the $\mathrm{eCHTC}_{w}$ distribution, as shown by Liu et al. (2015). A similar behaviour is observed for wind pressure coefficients (Costola et al. (2009)). BIPV modules in the building envelope may be particularly susceptible to $\mathrm{eCHTC}_{w}$ and pressure variations since local effects are even more important.

Following this rationale, three research questions have been identified: How variations in heat transfer and ventilation parameters affect the BIPV performance? Which inputs have the strongest influence? How does the eCHTC affect the BIPV behaviour?

This paper tries to address these questions through a sensitivity analysis (SA). SA provides insight on the model behaviour and its response to variations in the model inputs. Such results can provide quantitative information to guide the decision for future work, in particular, (1) to indicate which parameters are critical regarding heat transfer and natural ventilation effects, and (2) to decide upon the need to further assess the impact of the built environment on the performance of BIPV systems.

Several techniques for sensitivity analysis are available, each one having its specific characteristics (Saltelli et al. (2008)). The simplest sensitivity method, also called local or differential SA, varies the parameters one-at-a-time around a base case. Local $\mathrm{SA}$ is less computational intensive and allows an intuitive interpretation of the results. Drawbacks to this approach include the exploration of only a reduced input space, the impossibility of evaluating the interactions between inputs, and the lack of self-verification to explain how much of the output variation is due to the input variation. Such method has been applied to BIPV problems to investigate, for example, the influence of the distance between the BIPV module and the building wall (DeBlois et al. (2013); ElSayed (2016); Peng et al. (2016)) and the influence of the airflow rate in mechanically ventilated BIPV systems (Wilson and Paul (2011); Peng et al. (2016)).

Alternatively, global SA focuses on the influence of different inputs over the whole input space and are, in general, capable of overcoming the three limitations of local methods. Despite being an important resource for modelling methodologies, global SA has not been comprehensively explored for BIPV modelling, as highlighted by Cipriano et al. (2016). These authors applied a data-driven approach to validate a naturally ventilated PV component using experimental data. In their approach, a global SA method is employed to identify the unknown parameters and evaluate their influence on the model output. The coefficients related to the airflow and heat transfer as well as the optical properties of the BIPV module have been identified as strong parameters. Importantly, the external convection heat transfer coefficient $\left(\mathrm{eCHTC}_{w}\right)$ was not considered in the $\mathrm{SA}$ due to low wind intensity during the monitoring period.

In order to investigate the research questions introduced previously, this paper assesses in a systematic way the impact of different modelling parameters on the BIPV performance, including the $\mathrm{eCHTC}_{w}$. Different SA methods are applied with a design perspective, to understand the BIPV response in terms of power and operating temperature (outputs) to variations in the heat transfer and ventilation parameters (inputs). It is the research purpose that differs this work from the one by Cipriano et al. (2016), in which sensitivity and uncertainty analyses have been used to identify unknown parameters in an experimental validation exercise. The implications of this objective in terms of methodology will be addressed later.

After this introductory section, a brief description of the BIPV model is provided. Next, the methodology employed in the sensitivity analysis is presented. The subsequent two sections present and discuss the results. The last section summarises and concludes the paper.

\section{BIPV model}

The multi-physics BIPV model used in this work has been previously developed within the IDEAS environment, an open-source framework for building and district energy simulations (Jorissen et al. (2018)). The model has been validated for two different crystalline silicon (c-Si) BIPV modules, part of the southwest facade of the Vliet test building in Leuven, Belgium, with focus on temperature and energy yield predictions (Goncalves et al. (2018a, 2018b)).

In the present study, a c-Si BIPV module with a rated power of $244 \mathrm{Wp}$ is considered, as described by Goncalves et al. (2018b). This BIPV configuration presents a $15-\mathrm{cm}$ insulation layer separating the cavity from the interior environment. Such characteristic allows simplifying the modelling of the building interior, taking a convective heat transfer coefficient and a constant interior temperature of $21{ }^{\circ} \mathrm{C}$ as boundary condition. In fact, preliminary simulations have shown that, in this case, the indoor temperature has negligible effect on the performance indicators.

Figure 1 illustrates the object-oriented approach used in the BIPV model. Each PV element is combined to one thermal element, which encapsulates the heat transfer and airflow modelling, as well as the coupling to the building interior. The temperature-dependent one-diode model is employed to represent the electri- 


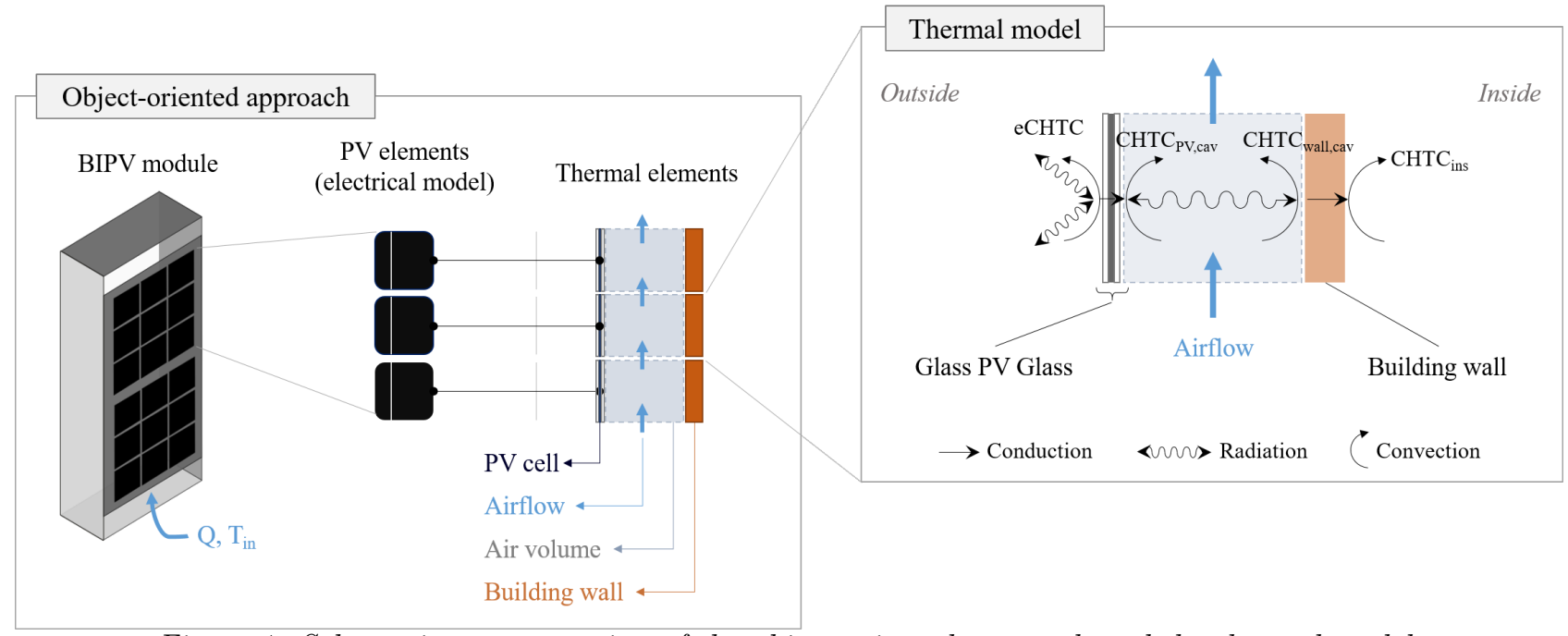

Figure 1: Schematic representation of the object-oriented approach and the thermal model.

cal behaviour. Each PV element corresponds to one PV cell and has a different solar irradiation intensity and temperature as input, allowing the simulation of shading effects as well as temperature gradients over the module. While shading effects are not included in the present work, the temperature gradient over the BIPV height due to buoyancy is considered.

A schematic representation of the thermal model, including both heat transfer and ventilation, is also shown in Figure 1. Conduction is treated as a onedimensional problem, assuming that the temperature is evenly distributed over the element surface. Material properties are considered homogeneous over the layer of each element and constant over time. Thermal bridges at the edges are neglected. Convection is based on correlations available in the literature. Detailed information can be found in Goncalves et al. (2018a; 2018b).

The relationship between the airflow rate, $Q$, and the pressure difference over a ventilated cavity, $\Delta \mathrm{P}$, is expressed as $\mathrm{Q}=\mathrm{C}_{d} \Delta \mathrm{P}^{n}$, where $\mathrm{C}_{d}$ is the flow coefficient, which represents the cavity (friction and turbulent) losses, and $\mathrm{n}$ is the flow exponent, which represents the flow regime (laminar or turbulent) (Straube and Burnett (1995)). In natural ventilation, $\Delta \mathrm{P}$ accounts for both buoyancy and wind effects.

\section{Methodology}

The SA methodology follows four steps. The design perspective adopted here requires a detailed explanation concerning the first two steps, i.e. the identification of the problem and the definition of the inputs and corresponding distributions. The third and fourth steps concerning the generation of input vectors and the SA methods are more straightforward steps, since they follow the methods well established in the literature.

\section{Identification of the problem}

Regarding the selection of inputs and outputs for sensitivity analysis, Tian (2013) explains that different research purposes ask for different decisions. The author takes the thermo-physical properties of the building envelope as example. These properties can be associated to a normal distribution if the goal is to compare the simulated performance of an existing building to its monitored behaviour. Normal distributions account for natural uncertainties, due to, for example, construction aspects, aging, and actual conditions of the building. Conversely, if the goal is to identify energy saving measures at the early design stage, these properties should be taken as equally probable and related to a uniform distribution. In this case, the materials composing the walls have not yet been decided; therefore, any value within a reasonable range would be possible.

The aim of this paper aligns with the second purpose, in which case the analyst should focus on the possible ranges for design variables, rather than natural uncertainties (normal distributions). Particular inputs may only be realistic for certain situations (e.g. rural or urban areas; high-rise or low-rise buildings), requiring a carefully interpretation of the results.

A second implication of the research purpose concerns the output for the sensitivity analysis. The outputs (or target functions) considered for the SA here are the power produced by the BIPV and the cell temperature at the top of the BIPV module, $\mathrm{T}_{B I P V}$, at an irradiance level of $1000 \mathrm{~W} / \mathrm{m}^{2}$ and ambient temperature of $25{ }^{\circ} \mathrm{C}$. The irradiance condition corresponds to the standard testing conditions (STC) for $\mathrm{PV}$ systems, providing a relevant reference to assess the BIPV performance. This choice is also independent from weather conditions or location and, therefore, generally valid (within the input ranges). 


\section{Definition of inputs and distributions}

This paper focuses on heat transfer and ventilation aspects and, therefore, only inputs related to these phenomena are considered for the SA. The following three CHTCs are considered (Figure 1): (1) between the BIPV front surface and the environment, eCHTC, which combines both wind and buoyancy effects; (2) between the BIPV back surface and the air inside the cavity, $\mathrm{CHTC}_{P V \text {,air }}$; and, (3) between the insulation/wall surface and the air inside the cavity, $\mathrm{CHTC}_{\text {wall,air }}$. As for ventilation, the three parameters that define the airflow rate are taken as input: (1) the airflow driving pressure difference, $\Delta \mathrm{P} ;(2)$ the flow coefficient, $\mathrm{C}_{d}$; and, (3) the flow exponent, $\mathrm{n}$. The temperature of the air entering the cavity, $\mathrm{T}_{i n}$, is the seventh input.

Defraeye et al. (2011) and Mirsadeghi et al. (2013) reviewed the existing correlations for eCHTC modelling. These studies show that eCHTC can reach values up to $100 \mathrm{~W} /\left(\mathrm{m}^{2} \mathrm{~K}\right)$ for wind speed between 0 and $15 \mathrm{~m} / \mathrm{s}$, depending on the correlation used. The eCHTC range has been defined to represent the most common values, between 0.3 and $50 \mathrm{~W} /\left(\mathrm{m}^{2} \mathrm{~K}\right)$. In terms of urban environment, low eCHTC values are representative of low-rise building and dense urban environment, while high values correspond to highrise buildings and rural areas.

As for natural convection inside cavities, the survey presented by Agathokleous and Kalogirou (2016) indicates that $\mathrm{CHTC}$ values are expected to be in range from 1 to $15 \mathrm{~W} /\left(\mathrm{m}^{2} \mathrm{~K}\right)$. The limits for $\mathrm{CHTC}_{P V \text {,air }}$ and $\mathrm{CHTC}_{\text {wall,air }}$ have been chosen to correspond to this range. The experimental study conducted by La Pica et al. (1993) indicates that values around $5 \mathrm{~W} /\left(\mathrm{m}^{2} \mathrm{~K}\right)$ are realistic for natural convection in cavities. Higher values may represent enhanced heat transfer techniques, such as fins or heat pipes.

Different authors have obtained the ventilation driving pressure in various building elements using experimental data (Saelens and Hens (2001); Falk and Sandin (2013); Langmans and Roels (2015)). In all these works, the magnitude of the driving pressure is similar, and typically below $2 \mathrm{~Pa}$. According to Straube and Burnett (1995), driving pressures in natural ventilation can be expected to be in the order of 1 Pa. Because the driving pressure depends on several parameters, a wider range has been defined, from 0.1 to $2 \mathrm{~Pa}$. Here, the lower values may represent sheltered buildings, where only buoyancy drives the flow. Wind gusts may cause even higher pressure differences, but have not been considered due to its highly dynamic behaviour.

A flow exponent $n=0.5$ indicates that the flow is completely turbulent, while an exponent of 1.0 defines a completely laminar flow. For practical building calculations, Nevander and Elmarsson (1994) (apud Gudum and Rode (2003)) suggests the use of $n=0.7$.
Based on experiments of steady state flow through orifices, Straube and Burnett (1995) conclude that an exponent of 0.55 fits fairly well for different orifice sizes, with larger exponents observed only for the very small ones. Exponents between 0.44 and 0.59 have been experimentally obtained for different ventilated building elements (Saelens and Hens (2001); Langmans and Roels (2015)). In this work, a range between 0.4 and 0.7 has been defined. The authors acknowledge that values below 0.5 are not theoretically possible, but can be experimentally obtained as result of experimental noise Straube and Burnett (1995).

Cavity characteristics of traditional building elements have been experimentally determined by different authors (Saelens and Hens (2001); Langmans and Roels (2015)). Depending on the cavity geometry, the flow coefficient $\mathrm{C}_{d}$ also varies over a large range, from very small values as it is the case of open head joints and small orifices $\left(\mathrm{C}_{d} \approx 8 \mathrm{~m}^{3} /\left(\mathrm{h} \cdot \mathrm{Pa}^{n}\right)\right)$ to large values occurring in well-ventilated cavities with large openings $\left(\mathrm{C}_{d} \approx 800 \mathrm{~m}^{3} /\left(\mathrm{h} \cdot \mathrm{Pa}^{n}\right)\right)$. A slightly larger range between 7 and 900 has been undertaken here.

Concerning the temperature of the air entering the cavity, Saelens et al. (2004) have shown that, for double skin facades, inlet temperatures are notably higher (up to $15 \mathrm{~K}$ ) compared to the exterior temperatures. Measurements in the BIPV setups used to validate the BIPV model also indicate that the inlet temperature can be significantly higher than the ambient temperature. This temperature raise is due to local heat transfer occurring at the openings, which depends on the solar radiation and the cavity geometry. In this study, the inlet temperature range has been taken up to $10 \mathrm{~K}$ higher than the ambient temperature; thus, between 25 and $35^{\circ} \mathrm{C}$.

Table 1 lists the inputs for the SA and their respective minimum and maximum values. As explained previously, since this paper applies SA with a design perspective, a uniform distribution has been assigned to all the inputs. This means that any value within the defined ranges is equally probable. In this way, any combination between the inputs is possible, even if not realistic, and particular BIPV configurations or urban environment are not favoured.

Table 1: Inputs and corresponding ranges.

\begin{tabular}{c|c|c|c} 
Input & Min & Max & Unit \\
\hline $\mathrm{eCHTC} \mathrm{CHTC}_{P \text {, air }}$ & 0.3 & 50 & $\mathrm{~W} /\left(\mathrm{m}^{2} \mathrm{~K}\right)$ \\
$\mathrm{CHTC}_{\text {wall,air }}$ & 1 & 15 & $\mathrm{~W} /\left(\mathrm{m}^{2} \mathrm{~K}\right)$ \\
$\Delta \mathrm{P}$ & 0.1 & 2 & $\mathrm{~W} /\left(\mathrm{m}^{2} \mathrm{~K}\right)$ \\
$\mathrm{C}_{d}$ & 7 & 900 & $\mathrm{~Pa}$ \\
$\mathrm{n}$ & 0.4 & 0.7 & - \\
$\mathrm{T}_{\text {in }} /\left(\mathrm{h} \cdot \mathrm{Pa}^{n}\right)$ \\
\hline
\end{tabular}




\section{Generation of the input vectors}

The third step of the methodology is to generate the input vectors, which can be done through the various sampling techniques available in the literature (Saltelli et al. (2008)). Sobol samples $(\mathrm{N}=2000)$ have been used for the generation of scatter plots and the calculation of regression coefficients (explained below). Other SA methods are associated to specific sampling techniques, as it is the case of EFAST (extended Fourier amplitude sensitivity testing). For EFAST, 7000 samples have been generated using the sampling scheme described by Saltelli et al. (1999).

\section{SA methods}

Tian (2013) classifies global methods in four groups: regression, screening-based, variance-based, and meta-model sensitivity analysis. This paper focuses on regression coefficients and the variance-based EFAST method. Scatter plots are also used to obtain visual insight on the results.

By quantifying the extent to which a given input changes the target output, regression methods are easy to compute and to interpret. A regression coefficient can describe both the strength and the direction of a relationship. Two commonly used correlation measures are the Pearson's correlation coefficient $(\mathrm{P})$, which evaluates the linear relationship between one input and the target output, and the Spearman's correlation coefficient $(\mathrm{S})$, which evaluates their monotonic relationship, linear or non-linear. While the linear relationship implies a constant variation rate between input and output, a monotonic behaviour means that the output never increases or never decreases with increasing the input.

EFAST belongs to the variance-based family and is suitable for both monotonic and non-monotonic models. This method associates the variability of the outputs to the inputs and has been described in detail by Saltelli et al. (1999). The main sensitivity measures are the first order (individual) and total effects. The individual effects account for the contribution of each input factor to the output variability. Total effects compute the total contributions to the output variance due to the corresponding input, which include both individual and higher-order effects from interactions among inputs. The difference between these measures corresponds, thus, to the effect of interactions among inputs.

\section{Results}

\section{Scatter plots and correlation coefficients}

Scatter plots are used as a starting point to explore the relationship between inputs and outputs. Figure 2 shows the scatter plots of $\mathrm{T}_{B I P V}$ and power for all inputs selected in the SA. Power values lie mostly between 180 and $240 \mathrm{~W}$; temperature values, between $40^{\circ} \mathrm{C}$ and $80^{\circ} \mathrm{C}$. A fairly strong non-linear relationship between eCHTC and both $\mathrm{T}_{B I P V}$ and power is identified. No evident correlation is observed between $\mathrm{T}_{B I P V}$ and power and the other inputs.

The regression results in Table 2 indicate that $\mathrm{T}_{B I P V}$ is highly correlated to eCHTC. With $\mathrm{P}=-0.903$, their relationship cannot be considered linear, but with $\mathrm{S}=-0.98$ it can be assumed monotonic. The negative sign means an inverse relationship between the two variables. The coefficients for $\mathrm{CHTC}_{P V \text {,air }}$ and $\mathrm{T}_{\text {in }}$ also suggest correlation, but with significantly lower values. The results are in agreement with the visual inspection of the scatter plots in Figure 2. Inconclusive p-values have been obtained for $\mathrm{CHTC}_{\text {wall,air }}$, $\Delta \mathrm{P}$ and $\mathrm{n}$, for both $\mathrm{P}$ and $\mathrm{S}$ coefficients. Similar correlation coefficients have been obtained for power, except that $\mathrm{S}$ becomes inconclusive also for $\mathrm{C}_{d}$.

\begin{tabular}{l|c|c} 
Table 2: Regression coefficients for & $T_{B}$ \\
Input & $\mathrm{P}$ & $\mathrm{S}$ \\
\hline eCHTC & -0.903 & -0.980 \\
CHTC $_{P \text {, air }}$ & -0.147 & -0.123 \\
CHTC $_{\text {wall, air }}$ & -0.022 & -0.017 \\
$\Delta \mathrm{P}$ & -0.031 & -0.029 \\
$\mathrm{C}_{d}$ & -0.078 & -0.069 \\
$\mathrm{n}$ & 0.001 & -0.001 \\
$\mathrm{~T}_{\text {in }}$ & 0.083 & 0.093 \\
\hline
\end{tabular}

\section{EFAST analysis}

Figure 3 presents the histogram plots obtained for the target outputs power and $\mathrm{T}_{B I P V}$; important statistical indicators are also included in the figure. Given the input space used for the SA, the average power value is $223 \mathrm{~W}$, about $9 \%$ lower than the $244 \mathrm{Wp}$ at STC. The corresponding average value for the $\mathrm{T}_{B I P V}$ is $50^{\circ} \mathrm{C}, 25 \mathrm{~K}$ above STC. Depending on the combination of inputs, the $\mathrm{T}_{B I P V}$ can reach values up to $109^{\circ} \mathrm{C}$; the power output can be as low as $163 \mathrm{~W}$, $33 \%$ lower compared to STC.

The indices that represent individual and total effects on $\mathrm{T}_{B I P V}$ are shown in Figure 4. Very similar results were observed for the power, but have been omitted due to space limitation. Besides, the heat transfer and ventilation inputs affect directly the $\mathrm{T}_{B I P V}$; the power determined as a function of $\mathrm{T}_{B I P V}$. The sum of all individual effects is less than 0.1 , which means that most of the output variability is due to interactions among the inputs rather than individual effects. Although eCHTC and $\mathrm{CHTC}_{P V \text {, air }}$ have the largest individual indices, together they account for only $13 \%$ of the output variability. Considering also their interactions with the other inputs, their influence rises to over $70 \%$ of output variability. Notably, $\mathrm{n}, \mathrm{C}_{d}$ and $\Delta \mathrm{P}$, which represent the cavity ventilation, become more important when interactions are considered. 


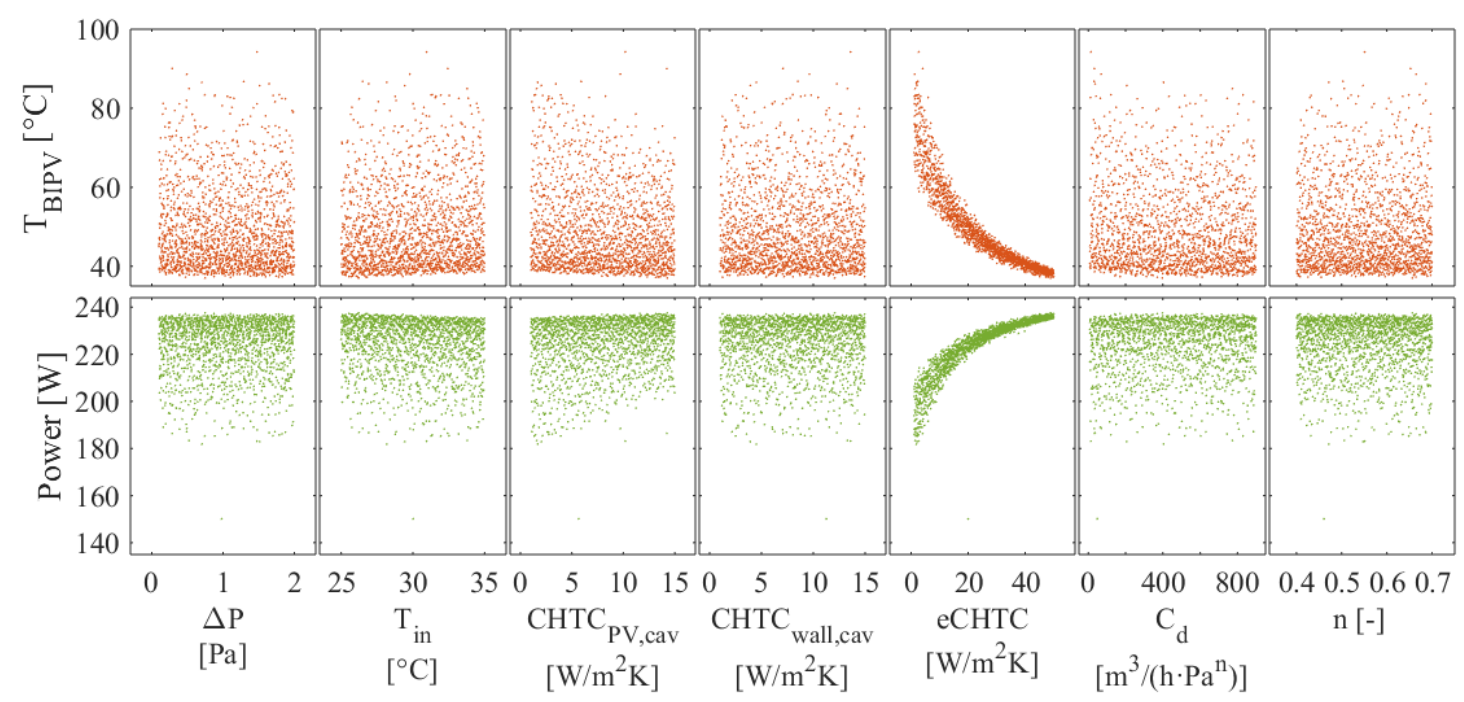

Figure 2: Scatter plots (Sobol samples, N=2000).

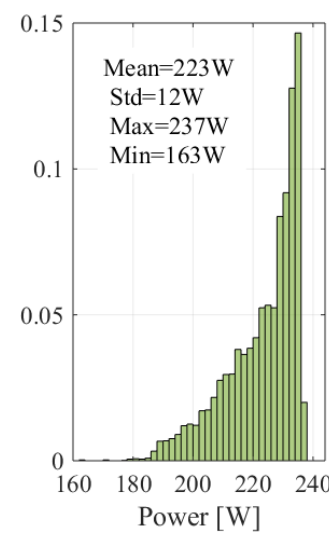

Figure 3: Power and $T_{B I P V}$ histogram distributions.

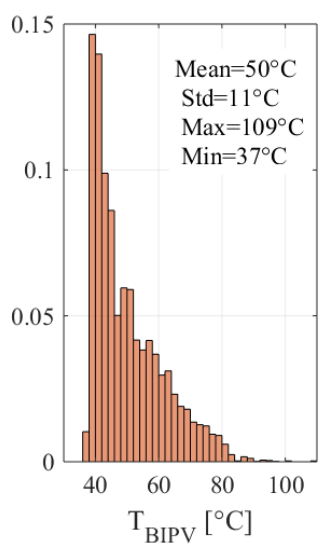

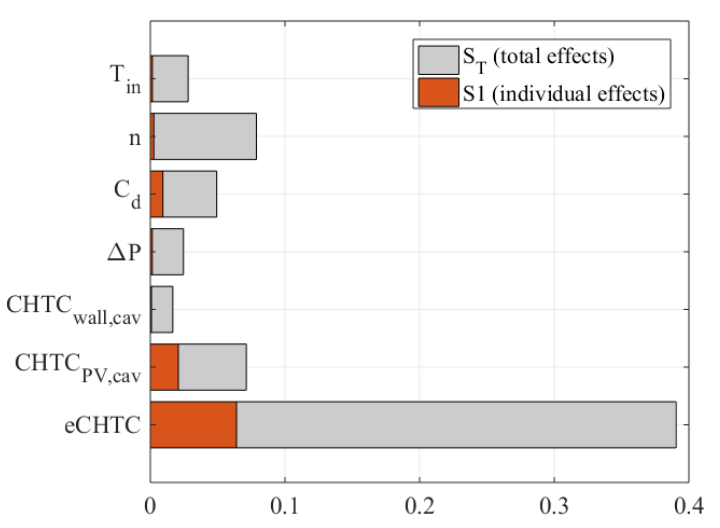

Figure 4: Individual and total effects on $T_{B I P V}$.

\section{Discussion}

Regarding heat transfer and ventilation aspects, the built environment imposes additional challenges to the modelling and optimisation of BIPV and BAPV systems. SA is an important aspect of modelling methodologies, providing insight on how the BIPV behaves when the inputs are changed. In this paper, the SA results indicate that, among the chosen inputs, only the eCHTC presents a defined relationship with both target functions. EFAST analysis indicate that the interactions among inputs is significantly more important than individual effects. Yet, eCHTC and $\mathrm{CHTC}_{P V \text {,air }}$ and their interactions account for more than $70 \%$ of the outputs variability. Ventilation parameters, i.e. $\mathrm{C}_{d}, \mathrm{n}$ and $\Delta \mathrm{P}$, present a relatively strong impact if considered as a group.

The scatter plots presented in Figure 2 show that the eCHTC effect is particularly strong at values above $20 \mathrm{~W} / \mathrm{m}^{2} \mathrm{~K}$. As eCHTC decreases, the spread in power and $\mathrm{T}_{B I P V}$ increases, indicating that the inter- actions with the other inputs (i.e. ventilation inputs) become more important. Higher eCHTCs can be associated to high wind speeds that occur, for example, in rural areas or at the edges of high-rise buildings in low density urban areas. On the contrary, lower eCHTCs represent lower wind speeds, dense building clusters, and/or low-rise buildings. These results suggest, therefore, that BIPV modules operating in lower eCHTCs conditions require better ventilation in order to operate at lower temperatures and produce the same amount of energy.

The interpretation of these results can be extended to the impact of the use of different correlations to model the eCHTC. As identified by Mirsadeghi et al. (2013), several correlations have been proposed in the literature for the eCHTC calculation. Figure 2 shows that both power and $\mathrm{T}_{B I P V}$ tend to a constant value as eCHTC value increase, particularly above $30 \mathrm{~W} / \mathrm{m}^{2} \mathrm{~K}$, which suggests that at high wind speeds the uncertainty in eCHTC models is less important. However, at lower wind speeds, the choice of model 
could lead to significant differences. These findings are also qualitatively valid for BAPVs.

Importantly, the SA results are intrinsically related to the range of parameters, the target function (yield vs power), and the environmental conditions (STC vs a day or a year). The input ranges have been defined to account for diverse design situations in terms of cavity characteristics as well as heat transfer conditions. The higher values of $\mathrm{CHTC}_{P V \text {, air }}$ may represent different techniques of improving the heat transfer, such as the use of fins or heat pipe configurations, while values around $5 \mathrm{~W} / \mathrm{m}^{2} \mathrm{~K}$ are realistic for cases of natural convection in vertical cavities, as experimentally determined by La Pica et al. (1993). Mechanical ventilation would require larger ranges for $\mathrm{CHTC}_{P V \text {, air }}$, $\mathrm{CHTC}_{\text {wall,air }}$, and $\Delta \mathrm{P}$.

From a practical perspective, modifications in one parameter may have consequences on the others. For example, the adoption of fins to enhance the heat transfer could increase the losses in the cavity, which in turn could be compensated by increasing the openings size. While the approach in this paper considers these combinations both feasible and equally probable (i.e. fins and larger openings, and no fins and smaller openings), detailed modelling techniques such as computational fluid dynamics (CFD) are more appropriate to investigate particular configurations. This effort is, however, beyond the scope of this paper, but is a direction for future studies.

The choice of the target function and the environment conditions will be discussed together, as they depend on each other in this particular study. Power, being an instantaneous indicator, is related to the conditions occurring at a given moment. Energy yield, on the contrary, depends on the transient conditions over a given period. Considering longer periods, days or a year, would not be consistent with the goal of this study, since most of the parameters used in the SA analysis depend on the weather conditions. In other words, annual simulations with constant CHTC values would not be realistic. In the approach followed by Cipriano et al. (2016), normal distributions have been assigned to the coefficients of CHTC correlations; the weather conditions are taken into account, and annual simulations are possible. However, this also assumes a fixed behaviour for the CHTC, which is not consistent with the design perspective adopted in the present paper.

\section{Conclusion}

A sensitivity analysis (SA) has been performed in this study to identify the dominant parameters on the performance of ventilated facade-BIPV systems. Parameters associated with ventilation and convective heat transfer have been taken as inputs to the SA, while power and the cell temperature at the top of the BIPV module as target outputs.

The interactions among inputs have been found to be significantly more important than any individual effect, with eCHTC and $\mathrm{CHTC}_{P V \text {,air }}$ and their interactions accounting for more than $70 \%$ of the outputs variability. From a modelling point of view, the effect of interactions between heat transfer and ventilation parameters cannot be neglected. Further implications of the SA findings are (1) eCHTC should be carefully considered in BIPV modelling, and (2) good ventilation conditions should be designed for BIPV modules operating at low eCHTC conditions, i.e. low wind speeds, dense clusters, and low-rise buildings.

The first suggestion for future work is to combine the ventilation inputs into a single one, the airflow rate, in order to provide more tangible recommendations in terms of BIPV design. Further research will also focus on different ranges for the inputs. Interesting information could be obtained for particular situations. For instance, if low eCHTC conditions are expected for a given location, a narrow range could be selected for this input, or if the designer considers the use of mechanical ventilation, the ranges for CHTC inside the cavity and $\Delta \mathrm{P}$ could be adjusted.

\section{References}

Agathokleous, R. A. and S. A. Kalogirou (2016). Double skin facades (DSF) and building integrated photovoltaics (BIPV): A review of configurations and heat transfer characteristics. Renewable Energy 89, 743-756.

Brito, M., S. Freitas, S. Guimarães, C. Catita, and P. Redweik (2017). The importance of facades for the solar PV potential of a mediterranean city using LiDAR data. Renewable Energy 111, 85-94.

Cipriano, J., G. Houzeaux, G. Mor, U. Eicker, J. Carbonell, and S. Danov (2016). Development of a dynamic model for natural ventilated photovoltaic components and of a data driven approach to validate and identify the model parameters. Solar Energy 129, 310-331.

Costola, D., B. Blocken, and J. Hensen (2009). Overview of pressure coefficient data in building energy simulation and airflow network programs. Building and Environment 44(10), 2027-2036.

DeBlois, J. C., M. M. Bilec, and L. A. Schaefer (2013). Design and zonal building energy modeling of a roof integrated solar chimney. Renewable energy 52, 241-250.

Defraeye, T., B. Blocken, and J. Carmeliet (2011). Convective heat transfer coefficients for exterior building surfaces: Existing correlations and CFD modelling. Energy Conversion and Management 52(1), 512-522. 
ElSayed, M. (2016). Optimizing thermal performance of building-integrated photovoltaics for upgrading informal urbanization. Energy \& Buildings 116, 232-248.

Falk, J. and K. Sandin (2013). Ventilated rainscreen cladding: Measurements of cavity air velocities, estimation of air change rates and evaluation of driving forces. Building and Environment 59, 164-176.

Goncalves, J., G. Reynders, J. Lehmann, and D. Saelens (2018a). Modelling of a naturally ventilated BIPV system for building energy simulations. In International Building Physics Conference, 2018.

Goncalves, J., K. Spiliotis, J. Lehmann, K. Baert, J. Driesen, and D. Saelens (2018b). Experimental validation of a BIPV curtain wall model for building energy simulations. In Advanced Building Skins Conference, 2018.

Gudum, C. and C. Rode (2003). Moisture Transport and Convection in Building Envelopes. Ph. D. thesis, Technical University of Denmark, Denmark.

Jorissen, F., G. Reynders, R. Baetens, D. Picard, D. Saelens, and L. Helsen (2018). Implementation and verification of the IDEAS building energy simulation library. Journal of Building Performance Simulation, 1-20.

La Pica, A., G. Rodono, and R. Volpes (1993). An experimental investigation on natural convection of air in a vertical channel. International journal of heat and mass transfer 36(3), 611-616.

Langmans, J. and S. Roels (2015). Experimental analysis of cavity ventilation behind rainscreen cladding systems: A comparison of four measuring techniques. Building \& Environment 87, 177-192.

Liu, J., M. Heidarinejad, S. Gracik, and J. Srebric (2015). The impact of exterior surface convective heat transfer coefficients on the building energy consumption in urban neighborhoods with different plan area densities. Energy $\&$ Buildings 86, 449-463.

Mirsadeghi, M., D. Costola, B. Blocken, and J. L. Hensen (2013). Review of external convective heat transfer coefficient models in building energy simulation programs: Implementation and uncertainty. Applied Thermal Engineering 56(1-2), 134-151.

Montazeri, H., B. Blocken, D. Derome, J. Carmeliet, and J. L. Hensen (2015). CFD analysis of forced convective heat transfer coefficients at windward building facades: influence of building geometry. Journal of Wind Engineering and Industrial Aerodynamics 146, 102-116.

Nevander, L. E. and B. Elmarsson (1994). Fukthandbok: praktik och teori. Svensk byggtjänst.
Osseweijer, F. J., L. B. Van Den Hurk, E. J. Teunissen, and W. G. van Sark (2018). A comparative review of building integrated photovoltaics ecosystems in selected european countries. Renewable and Sustainable Energy Reviews 90, 1027-1040.

Peng, J., D. C. Curcija, L. Lu, S. E. Selkowitz, H. Yang, and W. Zhang (2016). Numerical investigation of the energy saving potential of a semitransparent photovoltaic double-skin facade in a cool-summer mediterranean climate. Applied Energy $165,345-356$.

Saelens, D. and H. Hens (2001). Experimental evaluation of airflow in naturally ventilated active envelopes. Journal of Thermal Envelope and Building Science 25(2), 101-127.

Saelens, D., S. Roels, and H. Hens (2004). The inlet temperature as a boundary condition for multipleskin facade modelling. Energy \& Buildings 36(8), 825-835.

Saltelli, A., M. Ratto, T. Andres, F. Campolongo, J. Cariboni, D. Gatelli, M. Saisana, and S. Tarantola (2008). Global sensitivity analysis: the primer. John Wiley \& Sons.

Saltelli, A., S. Tarantola, and K.-S. Chan (1999). A quantitative model-independent method for global sensitivity analysis of model output. Technometrics $41(1), 39-56$.

Saretta, E., P. Caputo, and F. Frontini (2018). A review study about energy renovation of building facades with BIPV in urban environment. Sustainable Cities and Society.

Straube, J. and E. Burnett (1995). Vents, ventilation, and pressure moderation. University of Waterloo Building Engineering Group Report for Canada Mortgage and Housing Corporation, Ottawa, Ontario.

Thevenard, D. (2005). Review and recommendations for improving the modelling of building integrated photovoltaic systems. In Proceedings of the 9th IBPSA Conference, Montreal, Canada. Citeseer.

Tian, W. (2013). A review of sensitivity analysis methods in building energy analysis. Renewable and Sustainable Energy Reviews 20, 411-419.

Wilson, M. J. and M. C. Paul (2011). Effect of mounting geometry on convection occurring under a photovoltaic panel and the corresponding efficiency using CFD. Solar Energy 85(10), 2540-2550. 